\title{
MINERALIZACIÓN Y/O SOLUBILIZACIÓN DEL FÓSFORO CONTENIDO EN LODOS PROVENIENTES DE RESIDUOS SALMONÍCOLAS MARINOS BIOAUMENTADOS CON CEPAS DE Aspergillus niger Y Trichoederma pseudokoningi
}

(Mineralization and/or solubilization phosphorus content from salmon waste sludge marine bioincreased with strains Aspergillus niger and Trichoederma pseudokoningi)

\author{
Dante Pinochet, $\mathbf{T}^{1}$., Eduardo Valenzuela, $\mathrm{F}^{*}$., \\ Jorge Solís, B'., John Clunes, $\mathbf{M}^{1}$. \\ 1. Instituto de Ingeniería Agraria y Suelos, \\ Facultad de Ciencias Agrarias, Universidad Austral de Chile. \\ Casilla 567. Fono 632221239. \\ 2. Instituto de Bioquímica y Microbiología, \\ Facultad de Ciencias, Universidad Austral de Chile. \\ Casilla 167. Fono 632221296. \\ *Autor para correspondencia: evalenzu@uach.cl
}

RECIBIDO: 05 de Octubre de 2014 APROBADO: 02 de Diciembre de 2014

LOS AUTORES DECLARAN NO TENER CONFLICTO DE INTERESES

Palabras claves: Iodos de salmonicultura, Aspergillus, Trichodermas, Fósforo

Key Word: salmon sludge, Aspergillus, Trichodermas, phosphorus.

\section{RESUMEN}

Se evaluó un lodo salmonícola, proveniente de centros de cultivo marinos, con el objetivo de evaluar el aumento en la mineralización y/o solubilización del fósforo del lodo salmonícola tratado con la inoculación de cepas chilenas de hongos. El lodo fue pretratado mediante esterilización, pasteurización y sin pretratar. Posteriormente, fue inoculado con cepas nativas de Aspergillus niger y Trichoderma pseudokoningii, usando dos controles: sin adición de inóculo y otro, con la cepa control $A$. niger ANC. Estas muestras fueron incubadas por 60 días, determinándose el pH y la disponibilidad de fósforo (P) en los lodos pretratados. Luego de esta primera fase, las muestras fueron incubadas en tubos de lixiviación a temperatura controlada durante 6 semanas evaluando la cinética de mineralización de $\mathrm{P}$ de los lodos. Los pretratamientos aplicados al lodo aumentaron la dispo- nibilidad de P con la disminución del pH. La esterilización del lodo influyó en la colonización microbial, presentando la mayor colonización de las cepas inoculadas y la mayor liberación de $\mathrm{P}$ a la solución. El pretratamiento de esterilización inoculado con la cepa control $A$. niger presentó el mayor potencial de mineralización de $P$, siendo significativamente mayor a las cepas nativas y al control evaluado.

\section{ABSTRACT}

A salmon sludge from marine farming centers was evaluated with the aim to increase the mineralization and/or solubilization of their phosphorus (P) content through the inoculation of Chilean fungi strains. The sludge was pretreated through sterilization, pasteurization and without any pretreatment. After were inoculated with native strains of Aspergillus niger and Trichoderma pseudokoningii, 
using two controls: without inoculum and with inoculation with $A$. niger ANC. The samples were incubated for 60 days at room temperature and $\mathrm{pH}$ and $P$ availability was determined. After this first phase, the samples were additionally incubated for other 6 weeks, in leaching tubes, at controlled temperature $\left(25^{\circ} \mathrm{C}\right)$ evaluating the sludge kinetics of $P$ mineralization. The pretreatment applied to the sludge increased $\mathrm{P}$ availability with a $\mathrm{pH}$ value decline. The sludge sterilization had an influence on the microbial colonization, presenting high colonization of the inoculated strains and more $P$ was available. The pretreatment of sterilization inoculated with the control strain $A$. niger presented the biggest potential for mineralization of $P$, being significantly higher than the native strains and control evaluated in this study.

\section{INTRODUCCIÓN}

Uno de los desafíos globales para el desarrollo sustentable de la productividad de los recursos naturales ha sido evaluar las alternativas del uso de los residuos orgánicos (Gross et al., 2012). Los residuos orgánicos de la salmonicultura presentan una concentración relativamente alta de fósforo $(P)$, además de la presencia de otros nutrientes esenciales, lo que lo constituye en un recurso de posible utilización como biofertilizante en la agricultura (Teuber et al., 2007). De esta forma, Mazzarino et al. (1998) en residuos salmonícolas colectado del fondo de un lago en la Patagonia Argentina, muestra valores de $3 \mathrm{~g} \mathrm{~N}, 10,7 \mathrm{~g} P$ y 0,16 $\mathrm{g}$ K por cada $100 \mathrm{~g}$ de materia seca (MS). Por su parte, Pinochet et al. (2001), en una caracterización química de un sedimento de salmón recolectado bajo las balsa-jaula proveniente del lago Natri en la Región de los Lagos - Chile, determinaron valores de $0,7 \mathrm{~g} \mathrm{~N} ; 3,3 \mathrm{~g} P$ y $0,03 \mathrm{~g} \mathrm{~K}$ por cada $100 \mathrm{~g}$ de materia seca. Posteriormente, Pinochet et al. (2004), determinó valores de $0,7 \mathrm{~g} \mathrm{~N}$ y $1,4 \mathrm{~g} P$ por $100 \mathrm{~g}$ de materia seca para un lodo del lago Llanquihue Chile y $0,8 \mathrm{~g} \mathrm{~N}$ y 3,2 g P por $100 \mathrm{~g} \mathrm{MS}$ para sedimentos recolectados en el mar (Chinquihue, Región de los Lagos). Todos estos resultados muestran que el contenido de $P$ es relativamente alto, aunque su tasa de mineralización del $P$ fue muy baja (Pinochet et al., 2004). Por ello, se hace necesario estudiar aumentar la mineralización del
P orgánico contenido en los residuos, lo cual puede hacerse a través de la bio-aumentación de los residuos con microorganismos capaces de acelerar la mineralización. De esta forma, el objetivo de este estudio fue evaluar si la bioaumentación con microorganismos de las cepas de Aspergillus niger y Trichoderma pseudokoningii, aumenta la mineralización y/o solubilización del fósforo presente en el lodo.

\section{MATERIAL Y MÉTODOS}

La recolección de los residuos salmonídeos se realizó desde el fondo del lago Llanquihue - Chile, mediante un vehículo submarino manejado remotamente (ROV), el cual vía mangueras realizó el ascenso de los lodos de edad desconocida hasta piscinas flotantes; lugar en que se les adicionaron floculantes, para permitir la extracción de agua. Las muestras analizadas en este estudio correspondieron a la mezcla de las distintas zonas de recolección, las cuales fueron guardadas por 10 meses en contenedores de plástico.

Se realizó una caracterización química de los lodos donde se determinó Materia Seca, $\mathrm{pH}$, fósforo por colorimetría, aluminio (Al), potasio $(\mathrm{K})$, sodio $(\mathrm{Na})$, calcio $(\mathrm{Ca})$ y magnesio $(\mathrm{Mg})$ por espectrofotómetro de absorción, Nitrógeno total $(\mathrm{N})$ y Carbono total $(\mathrm{C})$ por el método de Walkley-Black (Sadzawka et al., 2006).

\section{Inoculación e incubación de lodos salmonicolas}

Para la preparación de los medios de cultivos (pretratamientos) se dispusieron de 108 botellas con $100 \mathrm{~mL}$ de caldo malta estéril; a cada botella se le adicionó $50 \mathrm{~mL}$ de lodo de mar (equivalente a $10 \mathrm{gr}$ b.m.s). De estas muestras de Lodo Caldo Malta (LCM), 36 botellas fueron esterilizadas en autoclave a $121{ }^{\circ} \mathrm{C}$ por 20 minutos (LCME), 36 botellas pasteurizadas a baño maría $75^{\circ} \mathrm{C}$ por 15 minutos (LCMP) y 36 botellas sin ningún pretratamiento (LCM).

Cada uno de estos pretratamientos sin inocular, fueron considerados medio de cultivo control. Por otro lado, la inoculación de los pretratamientos con la cepa Aspergillus niger 
(ANC) proveniente de Centraalbureau voor Schimmelcultures de Baarn, Holanda, fue considera inoculación control. El resto de los pretratamientos fueron inoculados con dos cepas nativas, Aspergillus niger (ANN) y Trichoderma pseudokoningii (TPN) aislados de un Typic Hapludand, serie Pelchuquín de la Región de Los Lagos (Valenzuela et al., 2002). Tanto los controles como los tratamientos fueron realizados por triplicado. Dichas botellas inoculadas fueron incubadas por 60 días a temperatura ambiente $(21$ $\pm 5^{\circ} \mathrm{C}$ ) en un agitador orbital (Lab-line modelo 3521) a 150 r.p.m.

Una vez finalizado cada tiempo de incubación, se procedió a filtrar el contenido de cada botella con un filtro de papel Whatman $N^{0} 1$. Del material filtrado se depositaron $10 \mathrm{~mL}$ de extracto en tubos de vidrio venoget, en duplicado, para posteriormente medir el fósforo soluble presente mediante el método de Murphy-Riley (Murphy \& Riley, 1962), $\mathrm{pH}$ al agua y el peso seco micelial. El duplicado del tiempo 60 no se filtró y pasó a una segunda fase.

\section{Tasas de mineralización de fósforo}

El lodo caldo malta (LCM) incubado e inoculado después de 60 días da inicio a la segunda etapa (nuevo tiempo 0). El contenido de cada una de las botellas, fue colocado sobre lana de vidrio y $20 \mathrm{gr}$ de arena de cuarzo en tubos de lixiviación de $50 \mathrm{~mL}$ e incubadas durante 6 semanas a $25 \pm 1$ ${ }^{\circ} \mathrm{C}$, en una cámara de incubación. La humedad fue controlada mediante un sellado superior con parafilm, con pequeñas perforaciones realizada con una aguja. El fósforo mineral presente, fue medido con el método de Murphy-Riley (Murphy \& Riley, 1962). Los valores de fósforo disponible fueron transformados con $\log _{10}$, para que sigan una distribución normal. Las diferencias entre medias fueron evaluadas con el test de Tukey usando el programa Statgraphics Plus. Los valores de las tasas de mineralización de fosforo fueron acumulados y analizados en función del tiempo a través de una regresión no lineal mediante la ecuación de Boltzmann sigmoidea. Los parámetros de esta ecuación fueron analizados mediante una prueba de $t$ utilizando el programa GraphPad Prism v. 4,0 .

\section{RESULTADOS Y DISCUSION}

Caracterización química del lodo de salmón marino

El contenido de N, P, Na, K y pH fueron similares a los reportados para lodos de mar por diversos autores. Sin embargo, el contenido de C, la relación $\mathrm{C} / \mathrm{N}, \mathrm{Al}, \mathrm{Ca}$ y $\mathrm{Mg}$ son diferentes a lo reportado por algunos autores, dependiendo de las características de colección, alimentación de peces, el manejo de los lodos y el tipo de aguas (Pinochet et al., 2001, 2004; Mazzarino et al., 1997, 1998; Laos et al., 2000; Axler et al., 1997; Naylor et al., 1999)

Tabla 1. Valores promedio y desviación estándar de la caracterización química del lodo salmonícola marino utilizado en este estudio.

\begin{tabular}{|ll|l|}
\hline Parámetro (b.m.s.) & & Lodo mar \pm d.e. \\
\hline Materia Seca & $\left(\mathrm{g} 100 \mathrm{~g}^{-1}\right)$ & $20,04 \pm 0,21$ \\
$\mathrm{Ph}$ & & $7,27 \pm 0,03$ \\
$\mathrm{C}$ & $\left(\mathrm{g} 100 \mathrm{~g}^{-1}\right)$ & $6,46 \pm 0,35$ \\
$\mathrm{~N}$ total & $\left(\mathrm{g} 100 \mathrm{~g}^{-1}\right)$ & $3,81 \pm 0,45$ \\
$\mathrm{P}$ total & $\left(\mathrm{g} 100 \mathrm{~g}^{-1}\right)$ & $2,05 \pm 0,12$ \\
$\mathrm{P}$ organic & $\left(\mathrm{g} 100 \mathrm{~g}^{-1}\right)$ & $0,17 \pm 0,05$ \\
Al & $\left(\mathrm{g} 100 \mathrm{~g}^{-1}\right)$ & $0,71 \pm 0,08$ \\
Relación C/N & & $1,7 \pm 0,2$ \\
$\mathrm{Na}$ & $\left(\mathrm{g} 100 \mathrm{~g}^{-1}\right)$ & $1,99 \pm 0,14$ \\
$\mathrm{~K}$ & $\left(\mathrm{~g} 100 \mathrm{~g}^{-1}\right)$ & $0,14 \pm 0,01$ \\
$\mathrm{Ca}$ & $\left(\mathrm{g} 100 \mathrm{~g}^{-1}\right)$ & $0,08 \pm 0,02$ \\
$\mathrm{Mg}$ & $\left(\mathrm{g} 100 \mathrm{~g}^{-1}\right)$ & $0,026 \pm 0,002$ \\
\hline
\end{tabular}

b.m.s. $=$ base materia seca. d.e. $=$ desviación estándar

El lodo de este estudio (Tabla 1) se caracteriza por ser bajo en el contenido de $\mathrm{C}$, rico en $\mathrm{N}$, bajo contenido de $\mathrm{K}$, con un valor de pH cercano a la neutralidad y con un alto contenido de $P$ mineral y un bajo contenido de P orgánico. Folke \& Kautsky (1989) señalan que el $P$ llega al sistema balsa-jaula en forma mineral de compuesto precipitado y solo parcialmente 
en forma orgánica, del cual solo un porcentaje cercano al $30 \%$ se libera hacia la forma de fosfato.

Variación del fosforo disponible y pH en el lodo caldo malta control (LCM) sin adición de cepas de hongo

El pretratamiento del medio de cultivo con adición de lodo influye en la colonización fúngica. Se asume que los pretratamientos de esterilización y pasteurización disminuyen la carga microbial nativa en el lodo, favoreciendo la colonización posterior por las cepas introducidas (Tyagi et al., 2005). Sin embrago, este pretratamiento puede inducir cambios en otras propiedades o condición química del lodo. La Figura $1 \mathrm{~A}$, muestra que el pretratamiento causó un efecto sobre la disponibilidad del $P$. Inmediatamente posterior al pretratamiento, se determinó que la mayor disponibilidad de $\mathrm{P}$ se presentó en las muestras LCM sin pretratar (LCMstrt), llegando a ser 1,9 veces mayor que el fósforo disponible en LCM pasteurizado (LCMpast) y 4,3 veces mayor que el contenido de $P$ disponible determinado en LCM esterilizado (LCMest).

Después de 60 días de incubación (Figura 1B), se presentó una situación inversa a la mostrada en el tiempo inicial (Figura 1A). Las muestras de LCM, presentaron valores 2,4 veces inferiores al contenido de $\mathrm{P}$ disponible presentado por las muestras LCMpast y 3,9 veces menores a las del pretratamiento LCMest. Por su parte, el pH presentó cambios con el pre- tratamiento (Figura 1A). Se observó que inmediatamente posteriormente al pretratamiento, la muestra LCM presentó el nivel de pH más bajo, seguido por el LCMpast y el control esterilizado LCMest que presentó el nivel de $\mathrm{pH}$ más alcalino de los tres controles. Al tiempo 60 el nivel de pH más alcalino de los controles lo presentó el LCM, seguido por el LCMpast y el LCMest, que presentó el nivel más bajo (Figura 2B). Dado el comportamiento de las muestras de LCM causado por el pretratamiento, se evaluó la relación entre el nivel de $\mathrm{pH}$ y la disponibilidad de $\mathrm{P}$ mostrando que mientras más alcalino fue el pH del medio, menor fue la disponibilidad de P (Figura 1A). Así, en las muestras de LCM se observó que la mayor disponibilidad de $\mathrm{P}$ estuvo asociada a un $\mathrm{pH}$ más bajo y que las muestras de LCMpast presentaron un valor intermedio de disponibilidad de $\mathrm{P}$, cuando el $\mathrm{pH}$ del medio se fue haciendo más alcalino, mientras que la menor disponibilidad de $\mathrm{P}$ fue en las muestras de LCMest, con los valores de $\mathrm{pH}$ más altos registrados en este estudio. De forma similar, después de 60 días de incubación (Figura 1B), la relación entre pH y disponibilidad de $\mathrm{P}$ se tiende a mantener, ratificado una relación inversa entre el $\mathrm{pH}$ y la disponibilidad de $P$ de las muestras. Kim et al. (1998), afirmaron que existe una relación clara entre una disminución del valor de pH y un incremento de la concentración de $P$ soluble en caldo malta. La explicación a estos resultados se debe a que en medios no tamponados, como los caldos de cultivo, el $P$ se combina

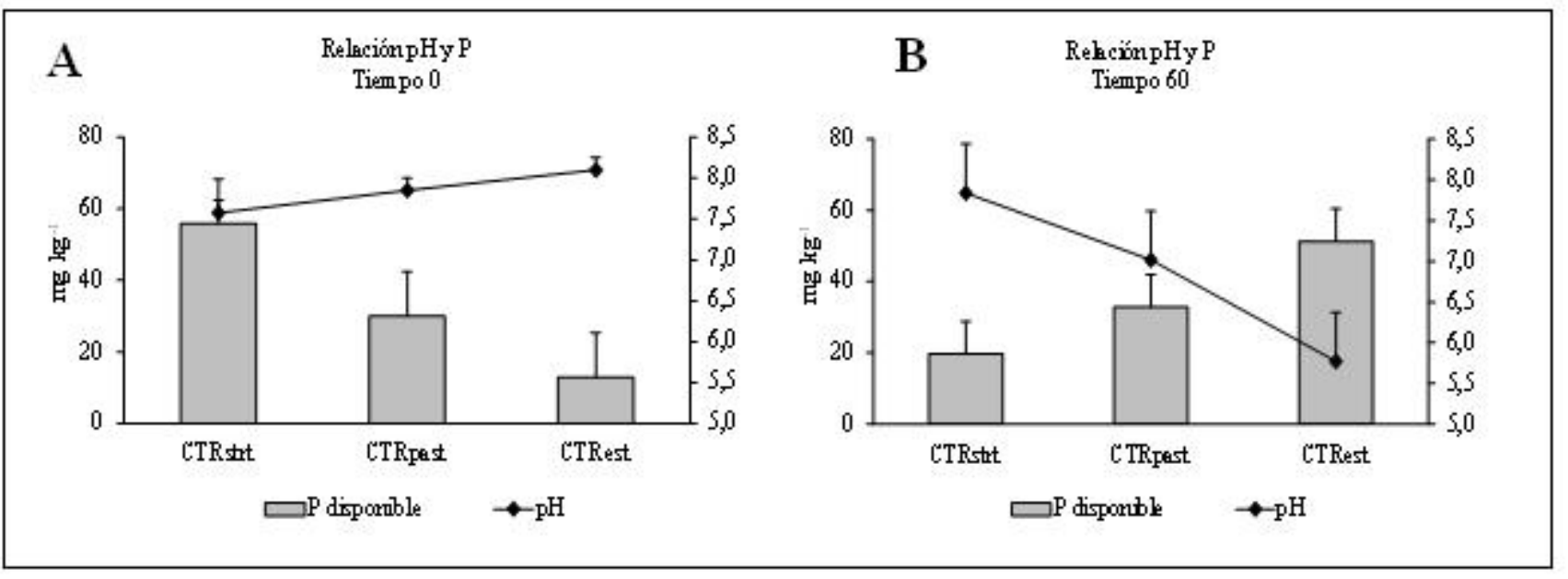

Figura 1. Relación del pH y P disponible para el lodo caldo malta control (LCMCTR).

A .En el tiempo cero. B. A los 60 días de incubación. 
con el calcio y el magnesio, formando sales insolubles, las cuales al hacer más ácido el medio se disocian y al no existir presencia considerable de Al u otro catión ácido en el medio, no se produce una precipitación del P como fosfato (Bohn et al., 1995).

\section{Variación del fósforo disponible y del pH con adición de cepas de hongos en los pretratamientos}

Después de 60 días de incubación de las muestras con los diferentes hongos se incrementó la disponibilidad del P (Figura 2) de ellas. Las muestras tratadas con A. niger control (ANC) fueron 3,2 veces superior al control LCM esterilizado sin adición de inóculo (CTR), seguido por las muestras tratadas con A. niger nativo (ANN) con 1,9 veces por sobre el nivel del CTR y por último las muestras tratadas con el hongo T. pseudokoningii nativo (TPN) cuyo aumento del nivel de $\mathrm{P}$ disponible no fue significativo con respecto al control.

A. niger presenta la habilidad de secretar ácidos orgánicos y/o exoenzimas, lo que produciría la solubilización de compuestos fosforados precipitados, principalmente fosfatos ligados a $\mathrm{Ca}$ (Valenzuela et al., 2002). De esta forma, a través de un mecanismo de solubilización de P por disminución del pH y una probable mineralización del $\mathrm{P}$ orgánico se explicaría el aumento en la disponibilidad del fósforo asociado a
Ios tratamientos inoculados con Aspergillus. Por su parte, la cepa de T. pseudokoningii, se caracteriza por su acción descomponedora de materiales orgánicos, principalmente compuestos lignocelulósicos (Cabeza et al., 2004). En este estudio, se muestra que este hongo no aumentó la disponibilidad de $\mathrm{P}$ en relación al tratamiento control sin inoculación, lo que sugiere que por el escaso contenido de $C$ del lodo de mar, no presentó una gran cantidad de $\mathrm{P}$ asociado a materiales orgánicos y que la destrucción potencial de compuestos lignocelulósicos por parte de $T$. pseudokoningii no produciría un aumento significativo del $\mathrm{P}$ mineralizado, al menos en lodos similares al utilizado en este estudio.

La variación de $\mathrm{pH}$ de las muestras inoculadas después de los 60 días de incubación (Figura 2) muestra que el tratamiento con la cepa TPN fue el más alcalino, diferenciándose del tratamiento control CTR. Ambos tratamientos con las cepas de $A$. niger, no mostraron un nivel de pH inferior al tratamiento control, ubicándose en un nivel intermedio de pH. Esto sugiere que el $\mathrm{P}$ liberado podría estar asociado más a la mineralización de P que a su solubilización, dado que para aumentar la solubilización de los fosfatos se requiere de una mayor disminución del valor de $\mathrm{pH}$, cosa que no ocurrí. Para evaluar la posibilidad que el aumento de $\mathrm{P}$ disponible estuviese asociado a la

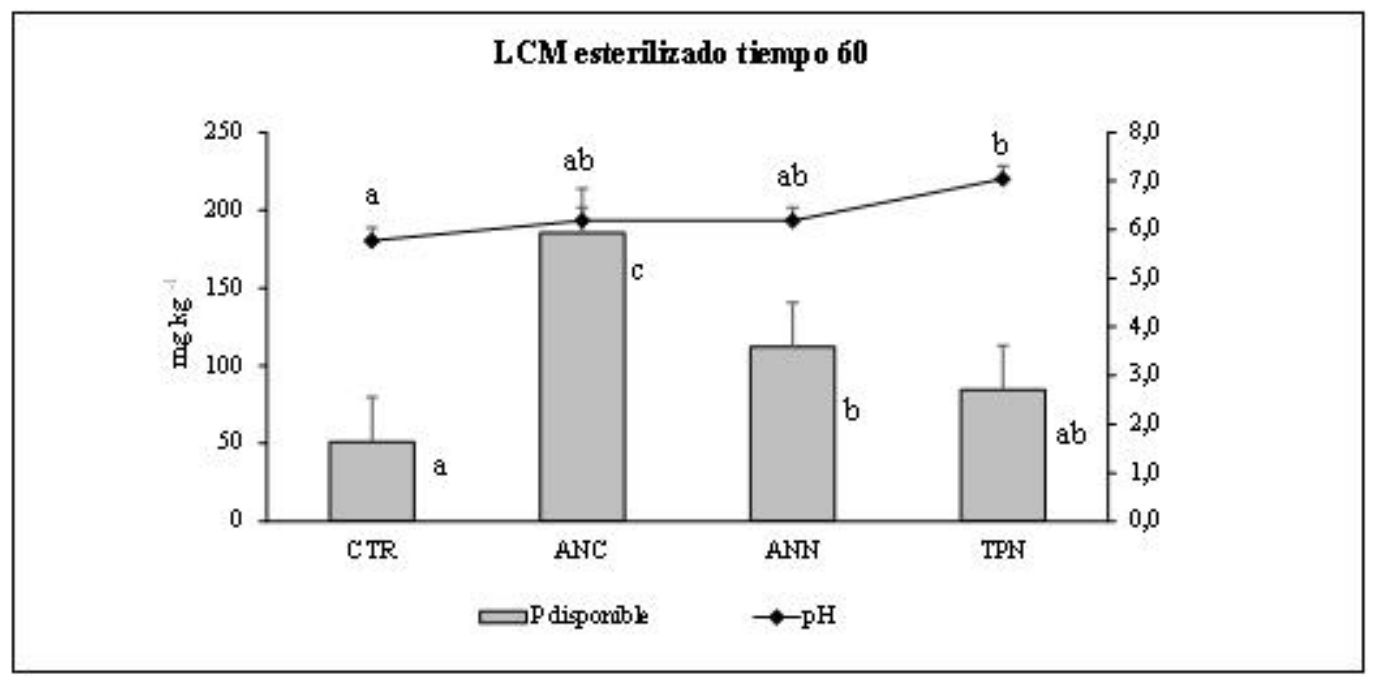

Figura 2. Nivel de $\mathrm{P}$ disponible y pH para el pretratamiento esterilizado, a los 60 días de incubación, del control (CTR), Aspergillus niger control (ANC), Aspergillus niger nativo (ANN) y Trichoderma pseudokoningii nativo (TPN). Letras distintas denotan diferencias significativas ( $p d » 0.05$ ). 
mineralización del P orgánico presente en el lodo, se requería determinar los mecanismos por lo cual ocurriría este proceso a través de la acción de fosfatasas, fitasas y fosfanatasas producidas por las cepas fúngicas (Rodriguez et al., 2006).

\section{Evaluación del crecimiento de los hongos a través del peso seco micelial}

Sólo en los tratamientos de LCM esterilizado después de 60 días de incubación fue posible determinar crecimiento micelial. De esta forma, para el pretratamiento de LCM esterilizado, luego de 60 días de incubación se determinó que la cepa A. niger control (ANC) presentó el mayor peso micelial (Tabla 2), seguida por la cepa nativa $A$. niger (ANN) que presentó un peso intermedio, siendo la cepa nativa T. pseudokoningii (TPN) la que presentó el menor peso micelial. Por lo tanto, el pretratamiento de pasteurización como el pretratamiento sin tratar, no permitieron el crecimiento de los hongos adicionados al sustrato, lo que sugiere que los microorganismos nativos presentes en el lodo y a los que quedaron después de la pasteurización dominaron por sobre los adicionados y que no habrían permitido su crecimiento $\mathrm{y} / 0$ desarrollo.

Los valores de peso micelial determinados en este estudio con el pretratamiento de esterilización son mayores a los determinados por Valenzuela et al. (2002) donde el peso del micelio de la cepa de $A$. niger a los 60 días fue cercano a $2 \mathrm{~g}$ base materia seca. Esto sugiere que para éste trabajo el crecimiento del micelio fue adecuado y permitió una buena colonización del sustrato, lo que habría ayudado a que se produjeran los efectos esperados de solubilización y mineralización del P contenido en el residuo.

Tabla 2. Peso del micelio seco en LCM esterilizado para la cepa Aspergillus niger control (ANC), y las cepas nativas Aspergillus niger (ANN) y Trichoderma pseudokoningii (TPN), a los 60 días de incubación.

\begin{tabular}{|c|c|c|}
\hline Tiempo & Hongo & Peso $(\mathbf{g})$ \\
\hline \multirow{3}{*}{60} & ANC & $5,573 \mathrm{~b}$ \\
& ANN & $4,417 \mathrm{ab}$ \\
& TPN & $4,067 . \mathrm{a}$ \\
\hline
\end{tabular}

Letras distintas denotan diferencia significativa $(P \leq 0.05)$

\section{Determinación de las curvas de mineralización de fósforo}

Al evaluar la mineralización, se consideró que el nivel de $P$ disponible determinado en el tiempo 60 de cada muestra pretratada, correspondió al nivel de P inicial de la mineralización. Para las curvas de mineralización este valor se descontó, haciéndolo igual a cero de modo de ajustarlo al tiempo cero. La mineralización de P mostró que el máximo correspondió siempre al tratamiento esterilizado, seguido del pasteurizado y después el sin tratar, independiente de que fuese con adición de cepa y del tipo de cepa de hongo (Tabla3). Estos resultados sugieren que la esterilización produjo un aumento en el potencial de mineralización al disminuir la carga bacteriana original, probablemente beneficiando la colonización de hongos (Tyagi et al., 2005). Por su parte, la pasteurización sólo disminuiría la carga bacteriana de forma que, inicialmente, se produce una rápida colonización de organismos presentes en el aire, pero que no fueron competitivos posteriormente para mantener la mineralización del sustrato.

Todos los tratamientos inoculados y el tratamiento sin inoculo presentaron una tasa de entrega creciente de P similar entre ellos. Estos resultados son relevantes, ya que descarta la hipótesis de este estudio que planteaba que las tasas de mineralización y/o solubilización serían mayores en Ios tratamientos inoculados con las cepas de A. niger y T. pseudokoningii, que con los tratamientos sin inoculación. De esta manera, para el LCM esterilizado el máximo potencial mineralizable fue de la cepa $A$. niger control (Figura 3a), para LCM pasteurizado se determinó en las muestras inoculadas con la cepas nativas $A$. niger y T. pseudokoningii (Figura 3b) y para LCM sin tratar lo mostró la cepa nativa T. pseudokoningii (Figura 3c), donde la muestra inoculada con TPN mostró una mejor adaptación al sustrato. Por otro lado, el tiempo de adaptación de los microorganismos al sustrato (V50) y el tiempo para desarrollar enzimas suficientes para comenzar a degradar este sustrato fueron similares para los distintos tratamientos, siendo también la tasa de entrega similar entre los ellos.

En la curva de Boltzmann el V50 representa el punto de inflexión, donde la curva se hace decreciente 
0 creciente. Su primera fase (creciente) hasta el V50, representa la etapa de adaptación de los microorganismos al sustrato. Esto es, antes que de que se produzcan enzimas suficientes para degradar el sustrato. Una vez establecidas las enzimas, en forma constante, se puede considerar que la reacción está dominada por el nivel de sustrato (Bohn et al., 1993) y está relacionada con los valores mostrados por sobre el punto de V50. Una relación entre el valor de V50 y la pendiente (en este caso 1/ Slope) muestra la relación entre la velocidad de adaptación microbial y el tiempo de adaptación microbial para producir las suficientes enzimas que permita la degradación del sustrato. La Figura 4 muestra los valores de relación entre V50 y la pendiente (1/Slope) de todas las ecuaciones ajustadas a los distintos pretratamientos y tratamientos del estudio de mineralización del lodo de mar evaluado.

La relación determinada fue negativa y significativa $(P<0.01)$ y al parecer en casi todos los puntos se cumple que la velocidad con que se alcanzó el punto de inflexión de la curva dependió de la adaptación microbial al sustrato, es decir la implantación de los hongos cultivados por sobre los microorganismos nativos del sustrato.

Tabla 3. Mineralización de fósforo en los tratamientos CTR sin inoculo, ANC, ANN y TPN con sus respectivos pretratamientos esterilizado, pasteurizado y sin tratar.

\begin{tabular}{|c|c|c|c|}
\hline \multicolumn{4}{|c|}{ Mineralización de Fosforo (parámetros de la ecuación Boltzmann) } \\
\hline \multicolumn{4}{|c|}{ Tratamiento CTR sin inoculo } \\
\hline $\begin{array}{l}\text { Esterilizado } \\
\text { Pasteurizado } \\
\text { Sin tratar }\end{array}$ & $\begin{array}{c}Y \max \\
70,2 \mathrm{~b} \\
60,1 \mathrm{a} \\
59,8 \mathrm{a}\end{array}$ & $\begin{array}{l}\text { V50 } \\
1,8 \mathrm{a} \\
1,7 \mathrm{a} \\
2,0 \mathrm{a}\end{array}$ & $\begin{array}{c}\text { slope } \\
0,56 \mathrm{~b} \\
0,32 \mathrm{a} \\
0,85 \mathrm{~b}\end{array}$ \\
\hline \multicolumn{4}{|c|}{ Tratamiento aspergillus niger control (ANC) } \\
\hline $\begin{array}{l}\text { Esterilizado } \\
\text { Pasteurizado } \\
\text { Sin tratar }\end{array}$ & $\begin{array}{l}Y \max \\
363,1 \mathrm{~b} \\
50,1 \mathrm{a} \\
46,9 \mathrm{a}\end{array}$ & $\begin{array}{l}\text { V50 } \\
1,3 \mathrm{a} \\
1,7 \mathrm{ab} \\
2,3 \mathrm{~b}\end{array}$ & $\begin{array}{l}\text { slope } \\
0,34 \mathrm{a} \\
0,59 \mathrm{ab} \\
0,87 \mathrm{~b}\end{array}$ \\
\hline \multicolumn{4}{|c|}{ Tratamiento Aspergillus niger nativo (ANN) } \\
\hline $\begin{array}{l}\text { Esterilizado } \\
\text { Pasteurizado } \\
\text { Sin tratar }\end{array}$ & $\begin{array}{l}Y \max \\
138,1 \mathrm{c} \\
90,3 \mathrm{~b} \\
52,7 \mathrm{a}\end{array}$ & $\begin{array}{l}\text { V50 } \\
1,4 \mathrm{a} \\
2,0 \mathrm{ab} \\
2,2 \mathrm{~b}\end{array}$ & $\begin{array}{c}\text { slope } \\
0,45 \mathrm{a} \\
0,86 \mathrm{a} \\
0,76 \mathrm{a}\end{array}$ \\
\hline \multicolumn{4}{|c|}{ Tratamiento Trichoderma pseudokoningii nativo (TPN) } \\
\hline $\begin{array}{l}\text { Esterilizado } \\
\text { Pasteurizado } \\
\text { Sin tratar }\end{array}$ & $\begin{array}{l}Y \max \\
81,2 \mathrm{~b} \\
92,1 \mathrm{~b} \\
65,0 \mathrm{a}\end{array}$ & $\begin{array}{l}\text { V50 } \\
1,1 \mathrm{a} \\
2,7 \mathrm{~b} \\
1,4 \mathrm{a}\end{array}$ & $\begin{array}{l}\text { slope } \\
0,39 \mathrm{a} \\
0,83 \mathrm{~b} \\
0,57 \mathrm{ab}\end{array}$ \\
\hline
\end{tabular}




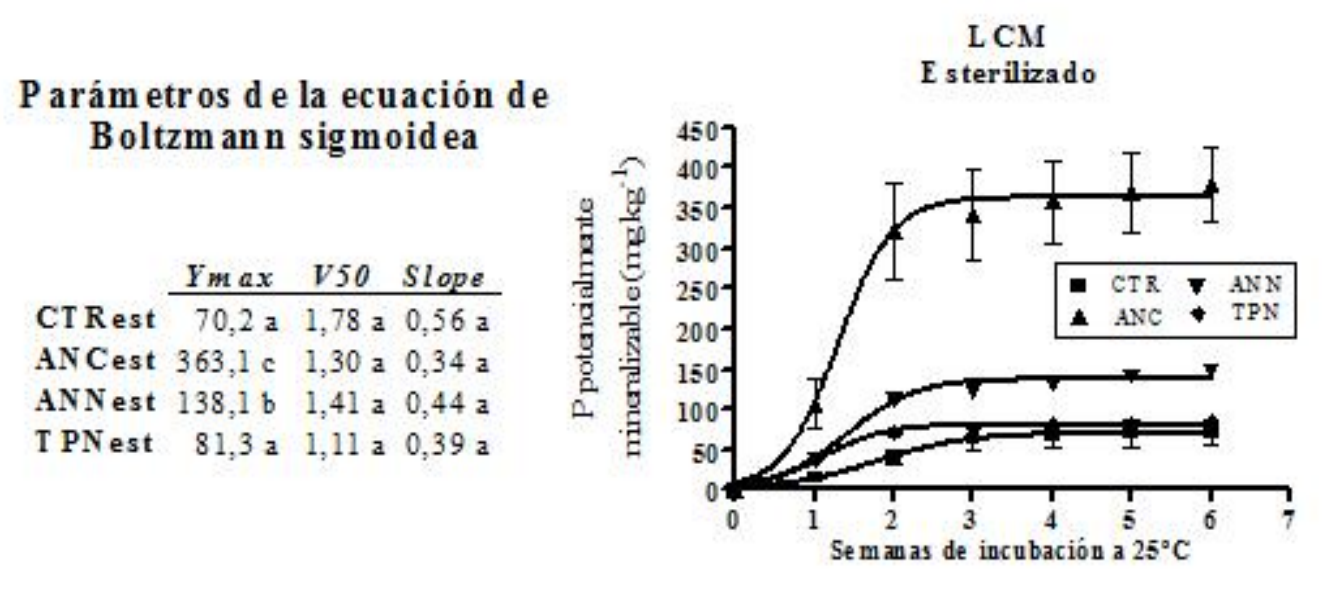

Parámetros de la ecuación de Boltzmann sigmoidea

\begin{tabular}{llll} 
& Ymax & V50 & Slope \\
\cline { 2 - 4 } CTR & $60,1 \mathrm{a}$ & $1,74 \mathrm{ab}$ & $0,32 \mathrm{a}$ \\
ANC & $50,1 \mathrm{a}$ & $1,71 \mathrm{a}$ & $0,59 \mathrm{a}$ \\
ANN & $90,4 \mathrm{~b}$ & $1,97 \mathrm{ab}$ & $0,86 \mathrm{a}$ \\
TPN & $92,1 \mathrm{~b}$ & $2,67 \mathrm{~b}$ & $0,83 \mathrm{a}$
\end{tabular}

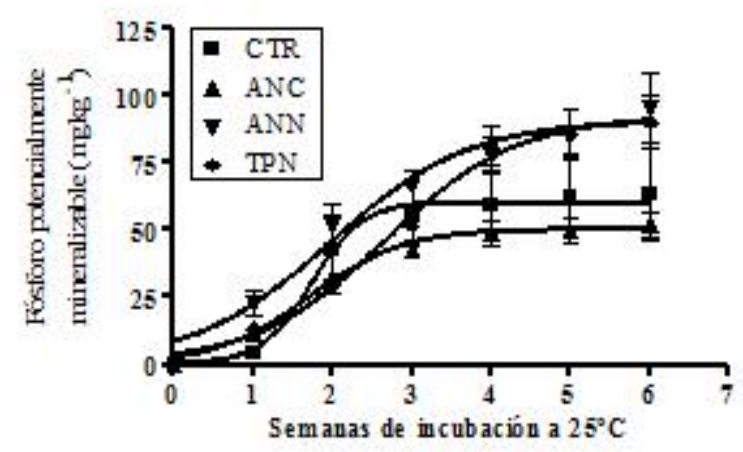

Parámetros de la ecuación de Boltzmann sigmoidea

\begin{tabular}{llll} 
& Ymax & V50 & Slope \\
\cline { 2 - 4 } CTR & $59,8 \mathrm{ab}$ & $2,0 \mathrm{ab}$ & $0,85 \mathrm{a}$ \\
ANC & $47,0 \mathrm{a}$ & $2,3 \mathrm{~b}$ & $0,87 \mathrm{a}$ \\
ANN & $52,7 \mathrm{ab}$ & $2,2 \mathrm{~b}$ & $0,76 \mathrm{a}$ \\
TPN & $65,0 \mathrm{~b}$ & $1,4 \mathrm{a}$ & $0,57 \mathrm{a}$
\end{tabular}
LCM

$\sin$ tratar

LCM

Pasteurizado

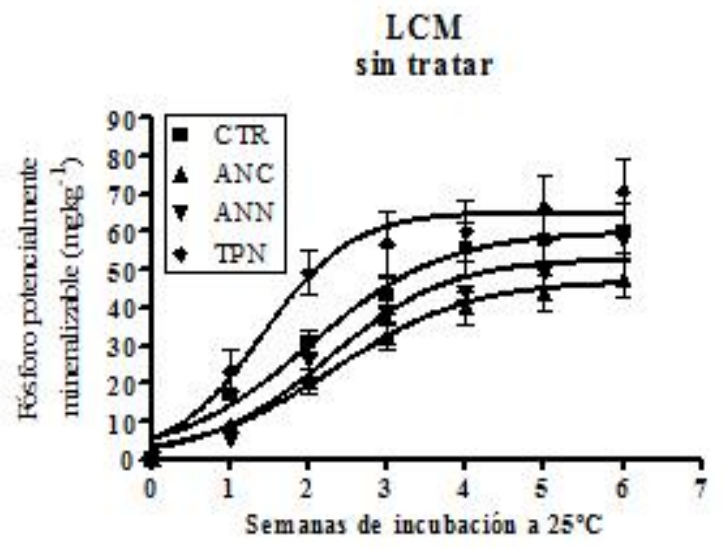

a

b

\section{b}

Figura 3. Curvas de acumulación de fósforo mineralizado y parámetros de la ecuación de Boltzmann para LCM esterilizado, LCM pasteurizado y LCM sin tratar. Letras distintas denotan diferencias significativas ( $p$ d» $0.05)$. 


\section{Ecuación lineal}

$$
\begin{aligned}
& Y=-1,11 x+3,84 \\
& r^{2}=0,52 \\
& S_{y x}=0,52 \\
& p<0.01
\end{aligned}
$$
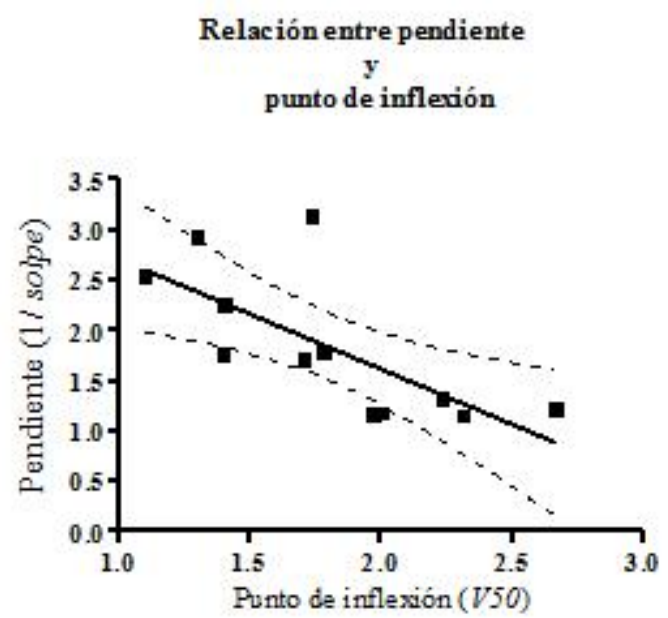

Figura 4. Relación entre la pendiente y el punto de inflexión de las curvas de mineralización.

\section{CONCLUSIONES}

El lodo evaluado en este estudio se caracterizó por un bajo contenido de $\mathrm{C}$, rico en $\mathrm{N}$, un alto contenido de $\mathrm{P}$ mineral y un bajo contenido de $\mathrm{P}$ orgánico. Además tuvo un bajo contenido de $\mathrm{K}$ y un valor de $\mathrm{pH}$ cercano a la neutralidad. Al esterilizar se influyó en la colonización microbial y en la disponibilidad de $\mathrm{P}$ en el medio de cultivo, presentando un mayor potencial de colonización de la cepa inoculada y el mayor potencial de liberación de P al medio de los pretratamientos aplicados en este estudio. Las tasas de mineralización de P no muestran diferencias de acuerdo al inóculo y el mayor potencial de mineralización de $\mathrm{P}$ lo presentó el tratamiento con esterilización inicial e inoculado con la cepa A. niger control, que fue diferente de la cepas nativas evaluadas en este ensayo.

\section{REFERENCIAS}

Axler, R., Tikkanen, C., Henneck, J., Schuldt, J. \& McDonald, M. (1997). Characteristics of effluent and sludge from two commercial rainbow trout in minnesota. The progressive fish - culturist. 59: 161-172.

Bohn, H., Mc Neal, B. \& O‘connor, g. (1995). Química de suelo. Ed. Limusa méxico $370 \mathrm{p}$.

Cabeza, R., Pinochet, D. \& Valenzuela, E. (2004). Biodegradación de paja de trigo por cepas fúngicas seleccionadas y su posterior incorporación a dos suelos de la décima región. Simposio de resíduos orgánicos y su uso en sistemas agroforestales. Sociedad chilena de la ciencia del suelo. Boletín: 20: 59-68.

Folke, C. \& Kautsky, N. (1989). The role of ecosystems for a sustainable development of aquaculture. Ambio. 18: 234-243.
Gross, A., Guy, 0., Posmanik, R., Fine, P. \& Nejidat, A. (2012). A novel method for combined biowaste stabilization and production of nitrate-rich liquid fertilizer for use in organic horticulture. Water air soil pollut. 223:1205-1214.

Kim, K., Jorda, D. \& Mc Donald, G. (1998). Effect of phosphate-solubilizing bacteria and vesiculararbuscular mycorrhizae on tomato growth and soil microbial activity. Biol. Fertil. Soils. 26: 79-87.

Laos, F., Satti, P., Walter, I., Mazzarino, M. \& Moyano, S. (2000). Nutrient availability of composted and noncomposted residues in a patagonian xeric mollisol. Biol. Fertil. Soils. 31: 462 - 469.

Mazzarino, M., Walter, I., Costa, G., Laos, F., Roselli, I. \& Satti, P. (1997). Plant response to fish farming wastes in volcanic soils. Journal of environmental quality. 26: 522-528. 
Mazzarino, M., Laos, F., Satti, P. \& Moyano, S. (1998). Agronomic and environmental aspects of utilization of organic residues in soils of the andean patagonian region. Soil science and plant nutrition. 44 (1):105-113.

Murphy, J. \& Riley h. (1962). A modified single solution method for the determination of phosphate in natural waters. Anal. Chim. Acta 27: 31-36.

Naylor, S., Moccia, R. \& Durant, G. (1999). The chemical composition of settleable solid fish waste (manure) from commercial rainbow trout farm in ontario, canada. North american journal of aquaculture. 61: 21-26.

Pinochet, D., Artacho, P. \& Azúa, P. (2001). Potencialidad como abono orgánico de los desechos sólidos subproducto del cultivo de especies salmonídeas. Agro sur. 29 (1): 78-82.

Pinochet, D., De Armas, M., Solis, J. \& Aymans, D. (2004). Tasas de mineralización para nitrógeno y fósforo en lodos de salmonicultura y sanitarios. Simposio de residuos orgánicos y su uso en sistemas agroforestales. Sociedad chilena de la ciencia del suelo. Boletín: 20: 59-68.

Rodríguez, H., Fraga, R., Gonzalez, T. \& Bashan, Y. (2006). Genetics of phosphate solubilization and its potencial applications for improving plant growthpromoting bacteria. Plant and soil. 287: 15-21.

Sadzawka, A., Carrasco, M., Grez, R., Mora, M., Flores, H. \& Neaman, A. (2006). Métodos de análisis recomendados para los suelos de chile revisión 2006. Instituto de investigaciones agropecuarias. Serie actas inia $n^{\circ}$ 34. Santiago. Chile. $164 \mathrm{p}$.

Teuber, N., Salazar, F., Alfaro, M. \& Valdebenito, A. (2007). Efecto de diferentes dosis de lodo de la crianza de salmones en el cultivo de papa y su efecto residual en ballica anual. Agricultura técnica. 67(4): 393-400.

Tyagi, R., Yezza, A., Valero, J. \& Surampalli, R. (2005). Production of bacillus thuringiensis-based biopesticides in batch and fed batch cultures using wastewater sludge as a raw material. J. Chem. Technol. Biotechnol. 80:502-510.

Valenzuela, E., Barrera, S. \& Pinochet, D. (2002). Solubilización de roca fosfórica carolina del norte con cepas de aspergillus niger aisladas desde un suelo trumao. Boletín micológico.17: 81-88.

Valenzuela, E., Pinochet, D. \& Carias, M. (2002). Mycological characterization of an hapludand soil series under three management practices. Mycotaxon 81: 357-366. 